\title{
Parameter Estimation and Hybrid Lag Synchronization in Hyperchaotic Lü Systems
}

\author{
Qing Wei and Zuolei Wang \\ School of Mathematical Sciences, Yancheng Teachers University, Yancheng 224002, China \\ Correspondence should be addressed to Zuolei Wang; wangzuolei1971@163.com
}

Received 24 October 2013; Revised 19 February 2014; Accepted 26 February 2014; Published 30 March 2014

Academic Editor: Dibakar Ghosh

Copyright (C) 2014 Q. Wei and Z. Wang. This is an open access article distributed under the Creative Commons Attribution License, which permits unrestricted use, distribution, and reproduction in any medium, provided the original work is properly cited.

\begin{abstract}
The antiphase and complete lag synchronization of hyperchaotic Lü systems with unknown parameters is investigated. Based on the Lyapunov stability theory, the sufficient conditions for achieving hybrid lag synchronization are derived. The optimized parameter observers are approached analytically via adaptive control approach. Numerical simulation results are presented to verify the effectiveness of the proposed scheme.
\end{abstract}

\section{Introduction}

Chaos has been thoroughly studied over the past two decades for its "random" behavior and sensitive dependence on the initial conditions. Despite the complexity and unpredictability of chaotic behavior [1], it can be controlled and two chaotic systems can be synchronized [2]. Since Pecora and Carroll introduced a method to synchronize two identical chaotic dynamical systems [3], the synchronization of chaotic dynamical systems attracted much attention due to its theoretical challenge and potential application in secure communications, chemical reactions, biomedical science, social science, and many other fields [4-6]. Various synchronizations have been presented, such as complete synchronization (CS), phase synchronization (PS), lag synchronization (LS) or anticipated synchronization (AS), and generalized synchronization (GS) [7-16]. Subsequently, many effective synchronization methods have been proposed, such as linear or nonlinear feedback synchronization, adaptive synchronization, lag synchronization, Q-S synchronization, and anticipated synchronization [17-26].

It is an interesting problem that part of the states of the interactive chaotic system are synchronized in one type of synchronization while other states synchronized in another type of synchronization. This phenomenon is taken as mixed synchronization. Due to the potential applications of it, some types of mixed synchronization are introduced recently. In [27], some variables may converge into synchronization while other variables are in antisynchronization state in Chen-Lee chaotic systems. In [28], using a scalar coupling, some of the state variables may be in complete synchronization while others may be in antisynchronization state in two unidirectionally coupled chaotic oscillators. It is found that lag synchronization has important technological implications. Generally, lag synchronization can be trivially accomplished by coupling the response system to a past state of the drive system or by mismatching of the system parameters. Therefore, inspired by $[29,30]$, it is invited to investigate the coexistence lag synchronization of chaotic systems via linear input both for theoretical research and practical applications. Mixed lag synchronization in the hyperchaotic systems could also be a useful topic, particularly for its potential application in secure communication due to its safety against attack and unmasking, the secure keys and carrier wave could be generated in more complex but safe way.

In this paper, the coexistence lag synchronization of two identical hyperchaotic Lü systems will be achieved only by linear controllers. Based on the Lyapunov stability theory, the sufficient conditions for achieving hybrid lag synchronization are derived. Numerical simulation results are presented to demonstrate the effectiveness of the proposed scheme. 


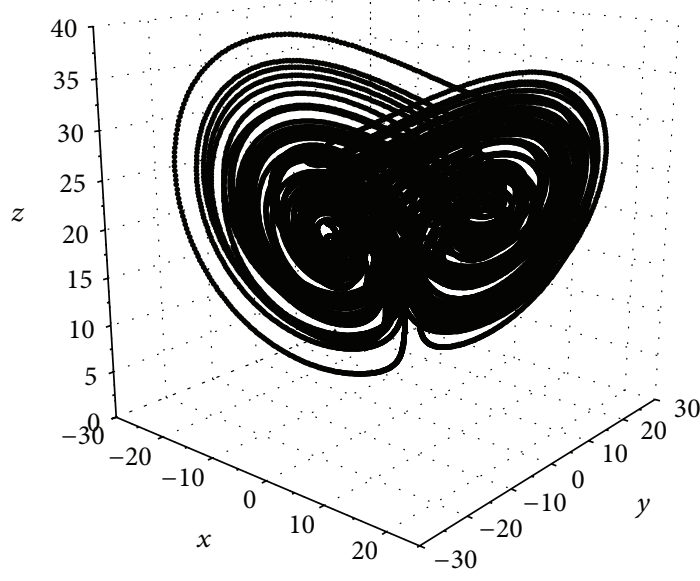

(a)

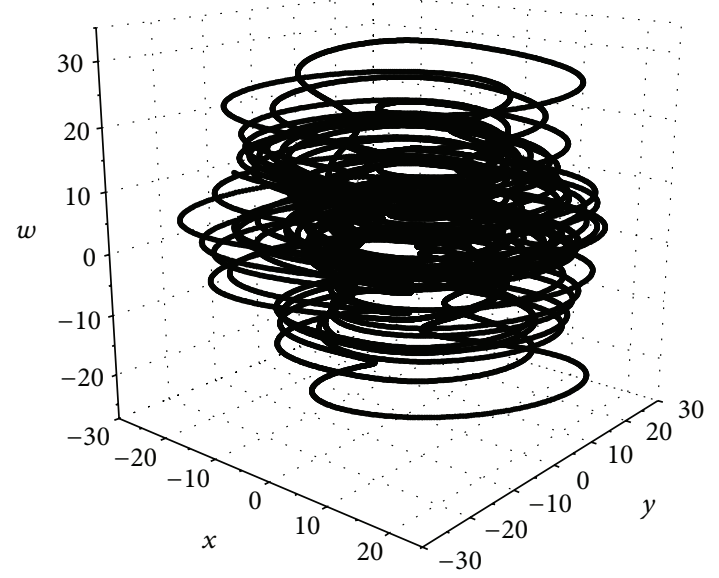

(b)

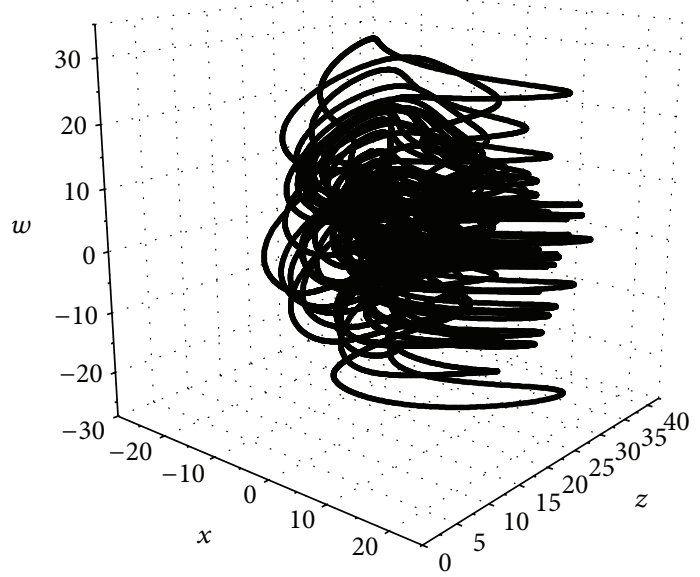

(c)

FIGURE 1: The hyperchaotic attractors of system (1): (a) $(x, y, z)$; (b) $(x, y, w)$; (c) $(x, z, w)$.

\section{System Description and Problem Formulation}

2.1. Hyperchaotic Lü System. In this paper, we consider the following hyperchaotic Lü system:

$$
\begin{gathered}
\dot{x}=a(y-x) \\
\dot{y}=c y+w-x z \\
\dot{z}=x y-b z \\
\dot{w}=-r_{1} x-r_{2} y .
\end{gathered}
$$

The dynamical behavior and its control have been studied in [31]. When $a=36, b=3, c=20, r_{1}=r_{2}=2$, system (1) has two positive Lyapunov exponents, that is, $\lambda_{1}=1.4106$, $\lambda_{2}=0.1232$, and the hyperchaotic attractors of system (1) are shown in Figure 1 (3D overview).

2.2. Hybrid Lag Synchronization Formulation. In this section, the hybrid lag synchronization of hyperchaotic Lü system will be investigated via linear feedback control method. For convenience, the drive system is denoted as the following:

$$
\begin{gathered}
\dot{x}_{m}=a\left(y_{m}-x_{m}\right) \\
\dot{y}_{m}=c y_{m}+w_{m}-x_{m} z_{m} \\
\dot{z}_{m}=x_{m} y_{m}-b z_{m} \\
\dot{w}_{m}=-r_{1} x_{m}-r_{2} y_{m} .
\end{gathered}
$$

The controlled response system is written as

$$
\begin{gathered}
\dot{x}_{s}=\widehat{a}\left(y_{s}-x_{s}\right)+u_{1} \\
\dot{y}_{s}=\widehat{c} y_{s}+w_{s}-x_{s} z_{s}+u_{2} \\
\dot{z}_{s}=x_{s} y_{s}-\widehat{b} z_{s}+u_{3} \\
\dot{w}_{s}=-\widehat{r}_{1} x_{s}-\widehat{r}_{2} y_{s}+u_{4},
\end{gathered}
$$

where $u_{1}, u_{2}, u_{3}, u_{4}$ are controllers to be constructed.

The target of this paper is to determine the controllers, such that the state variables $x_{s}, y_{s}$, and $w_{s}$ in response 

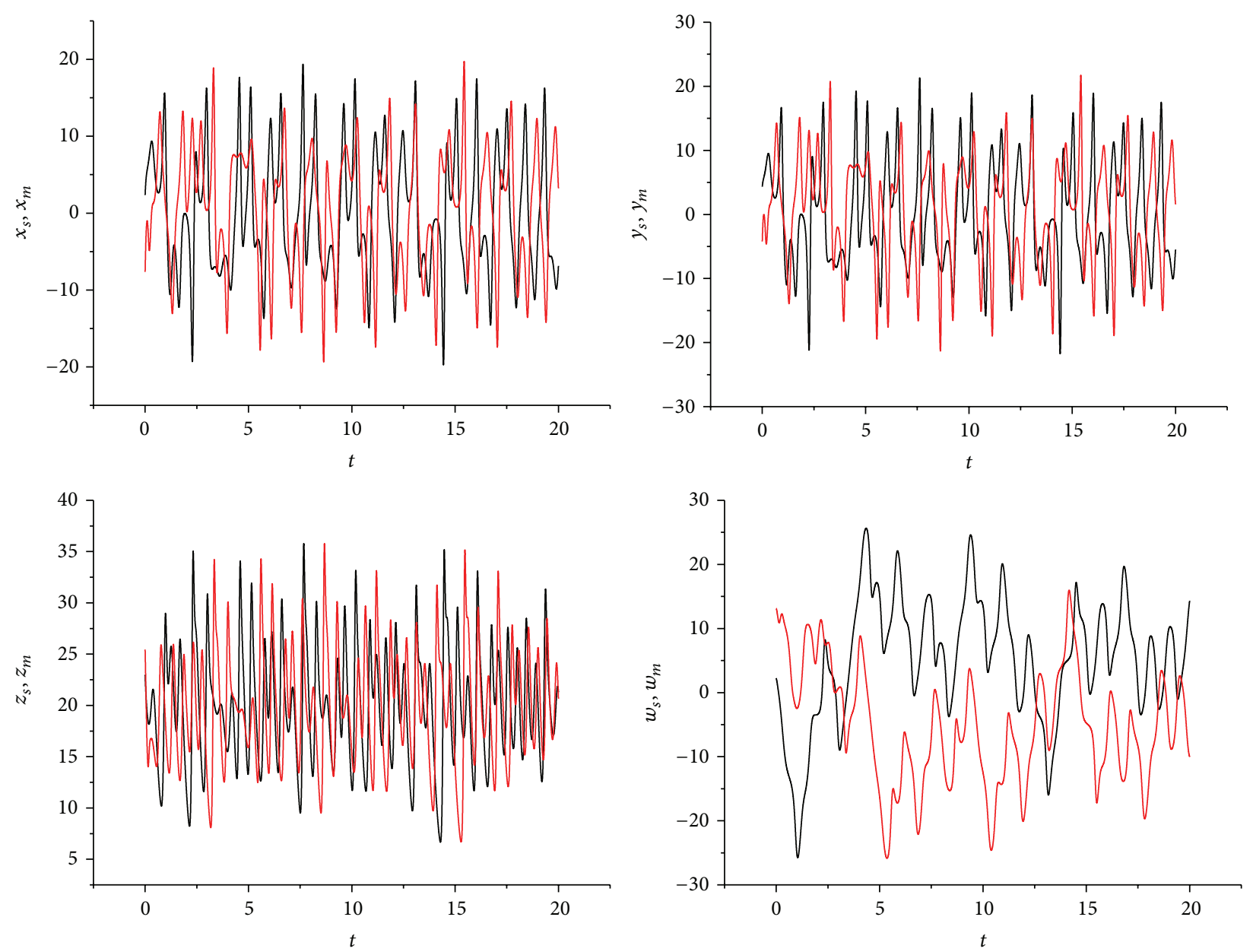

FIGURE 2: The evolutions of the state variables $x_{s}, y_{s}, z_{s}, w_{s}$ in response system and $x_{m}, y_{m}, z_{m}, w_{m}$ in drive system at $k=2.3$.

system antiphase synchronize to the $x_{m}, y_{m}$, and $w_{m}$ in drive system with a time lag, respectively, while the third state variable $z_{s}$ complete-synchronizes to $z_{m}$ with a time lag. For this purpose, the errors of corresponding variables of hybrid synchronization with a time lag and parameters are often defined as

$$
\begin{gathered}
e_{1}=x_{s}+x_{m}(t-\tau), \quad e_{2}=y_{s}+y_{m}(t-\tau), \\
e_{3}=z_{s}-z_{m}(t-\tau), \quad e_{4}=w_{s}+w_{m}(t-\tau), \\
e_{a}=a-\widehat{a}, \quad e_{b}=b-\widehat{b}, \quad e_{c}=c-\widehat{c}, \\
e_{r_{1}}=r_{1}-\widehat{r}_{1}, \quad e_{r_{2}}=r_{2}-\widehat{r}_{2} .
\end{gathered}
$$

Then we can get the following theorem.

Theorem 1. The third corresponding pair variables of systems (2) and (3) will reach complete lag synchronization, while other three pairs of corresponding variables of systems (2) and (3) will reach lag antisynchronization for any original values, when

$$
u_{i}=-k_{i} e_{i}, \quad(i=1,2,3,4)
$$

if the feedback coefficients $k_{i}(i=1,2,3,4)$ are large enough and the condition

$$
\begin{gathered}
\dot{e}_{a}=e_{1}\left(y_{s}-x_{s}\right) \\
\dot{e}_{b}=-e_{3} z_{s} \\
\dot{e}_{c}=e_{2} y_{s} \\
\dot{e}_{r_{1}}=-e_{4} x_{s} \\
\dot{e}_{r_{2}}=-e_{4} y_{s}
\end{gathered}
$$

is satisfied.

Proof. According to (4), the error system can be derived as

$$
\begin{gathered}
\dot{e}_{1}=a\left(e_{2}-e_{1}\right)-e_{a}\left(y_{s}-x_{s}\right)+u_{1} \\
\dot{e}_{2}=c e_{2}+e_{4}-x_{m}(t-\tau) z_{m}(t-\tau)-x_{s} z_{s}-e_{c} y_{s}+u_{2} \\
\dot{e}_{3}=x_{s} y_{s}-x_{m}(t-\tau) y_{m}(t-\tau)-b e_{3}+e_{b} z_{s}+u_{3} \\
\dot{e}_{4}=-r_{1} e_{1}-r_{2} e_{2}+e_{r_{1}} x_{s}+e_{r_{2}} y_{s}+u_{4} .
\end{gathered}
$$



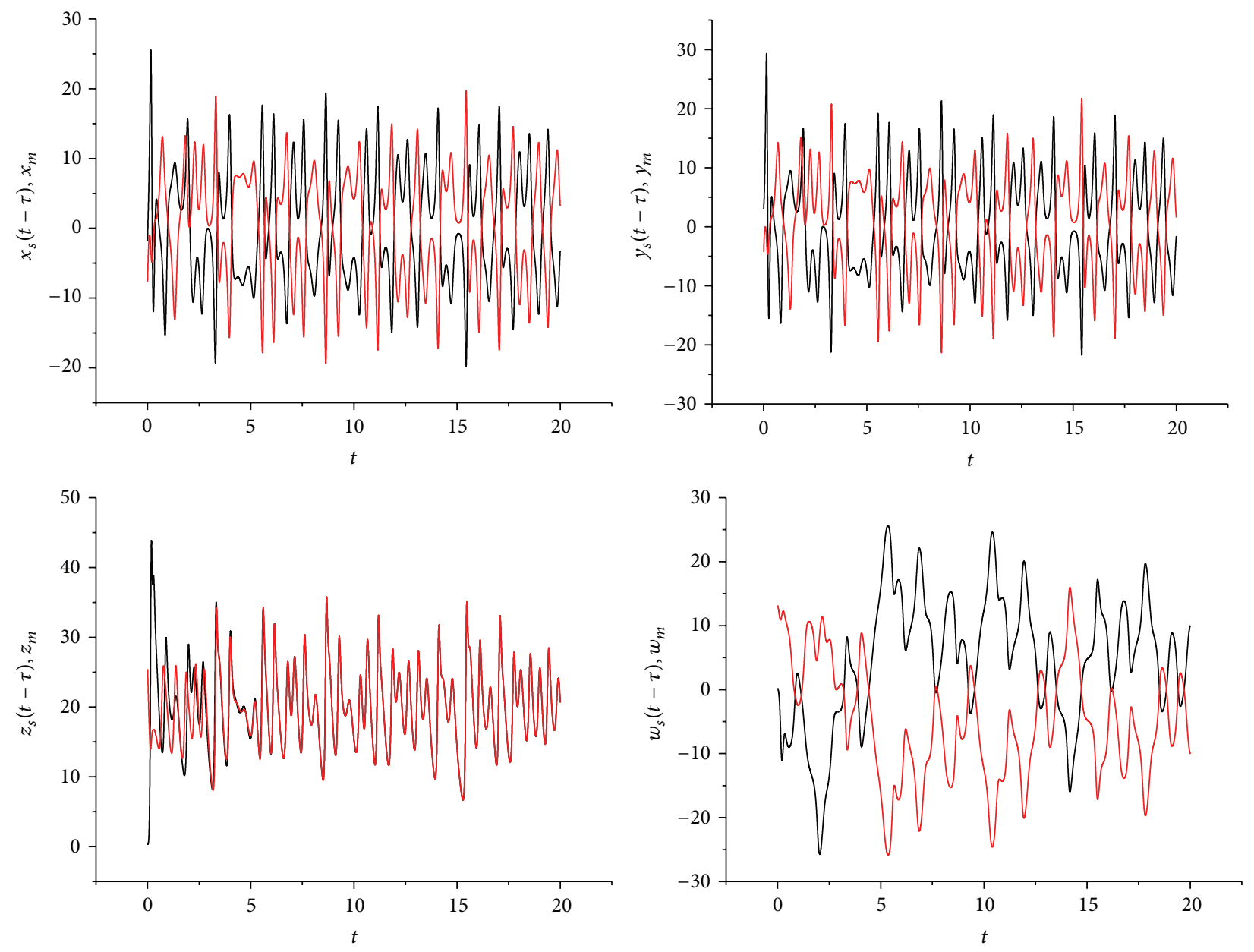

FIGURE 3: The evolutions of the state variables $x_{m}(t-\tau), y_{m}(t-\tau), z_{m}(t-\tau), w_{m}(t-\tau)$ and $x_{s}, y_{s}, z_{s}, w_{s}$.

Let Lyapunov stability function be

$$
V=\frac{\left[e_{1}^{2}+e_{2}^{2}+e_{3}^{2}+e_{4}^{2}+e_{a}^{2}+e_{b}^{2}+e_{c}^{2}+e_{r_{1}}^{2}+e_{r_{2}}^{2}\right]}{2}
$$

then

$$
\begin{aligned}
\dot{V}= & e_{1} \dot{e}_{1}+e_{2} \dot{e}_{2}+e_{3} \dot{e}_{3}+e_{4} \dot{e}_{4}+e_{a} \dot{e}_{a} \\
& +e_{b} \dot{e}_{b}+e_{c} \dot{e}_{c}+e_{r_{1}} \dot{e}_{r_{1}}+e_{r_{2}} \dot{e}_{r_{2}} \\
= & e_{1}\left[a\left(e_{2}-e_{1}\right)\right]+e_{a}\left[\dot{e}_{a}-e_{1}\left(y_{s}-x_{s}\right)\right]-k_{1} e_{1}^{2} \\
& +e_{2}\left[c e_{2}+e_{4}-x_{m}(t-\tau) e_{3}-z_{s} e_{1}\right]-k_{2} e_{2}^{2} \\
& +e_{c}\left[\dot{e}_{c}-e_{2} y_{s}\right]+e_{3}\left[e_{1} y_{s}-x_{m}(t-\tau) e_{2}-b e_{3}\right] \\
& +e_{b}\left[\dot{e}_{b}+e_{3} z_{s}\right]-k_{3} e_{3}^{2}+e_{4}\left(-r_{1} e_{1}-r_{2} e_{2}\right) \\
& +e_{r_{1}}\left[\dot{e}_{r_{1}}+e_{4} x_{s}\right]+e_{r_{2}}\left[\dot{e}_{r_{2}}+e_{4} y_{s}\right]-k_{4} e_{4}^{2} .
\end{aligned}
$$

Let $M$ be the bound positive value of hyperchaotic Lü system, that is, $\left|x_{m}\right|,\left|y_{m}\right|,\left|z_{m}\right|,\left|w_{m}\right|,\left|x_{s}\right|,\left|y_{s}\right|,\left|z_{s}\right|$, and $\left|w_{s}\right| \leq M$, which implies that

$$
\begin{aligned}
\dot{V} \leq & -\left(k_{1}-a\right) e_{1}^{2}-\left(k_{2}-c\right) e_{2}^{2}-\left(k_{3}-b\right) e_{3}^{2}-k_{4} e_{4}^{2} \\
& +(a+M)\left|e_{1}\right|\left|e_{2}\right|+M\left|e_{1}\right|\left|e_{3}\right|+r_{1}\left|e_{1}\right|\left|e_{4}\right| \\
& +2 M\left|e_{2}\right|\left|e_{3}\right|+\left(r_{2}+1\right)\left|e_{2}\right|\left|e_{4}\right| \\
= & -\left(\left|e_{1}\right|,\left|e_{2}\right|,\left|e_{3}\right|,\left|e_{4}\right|\right) P\left(\left|e_{1}\right|,\left|e_{2}\right|,\left|e_{3}\right|,\left|e_{4}\right|\right)^{T},
\end{aligned}
$$

where

$$
P=\left(\begin{array}{cccc}
k_{1}-a & -\frac{(a+M)}{2} & -\frac{M}{2} & -\frac{r_{1}}{2} \\
-\frac{(a+M)}{2} & k_{2}-c & -M & -\frac{\left(r_{2}+1\right)}{2} \\
-\frac{M}{2} & -M & k_{3}-b & 0 \\
-\frac{r_{1}}{2} & -\frac{\left(r_{2}+1\right)}{2} & 0 & k_{4}
\end{array}\right) .
$$

It is obvious that, for suitable values of $k_{i}(i=1,2,3,4)$, the matrix $P$ is positive definite and $\dot{V}$ is negative semidefinite. So 

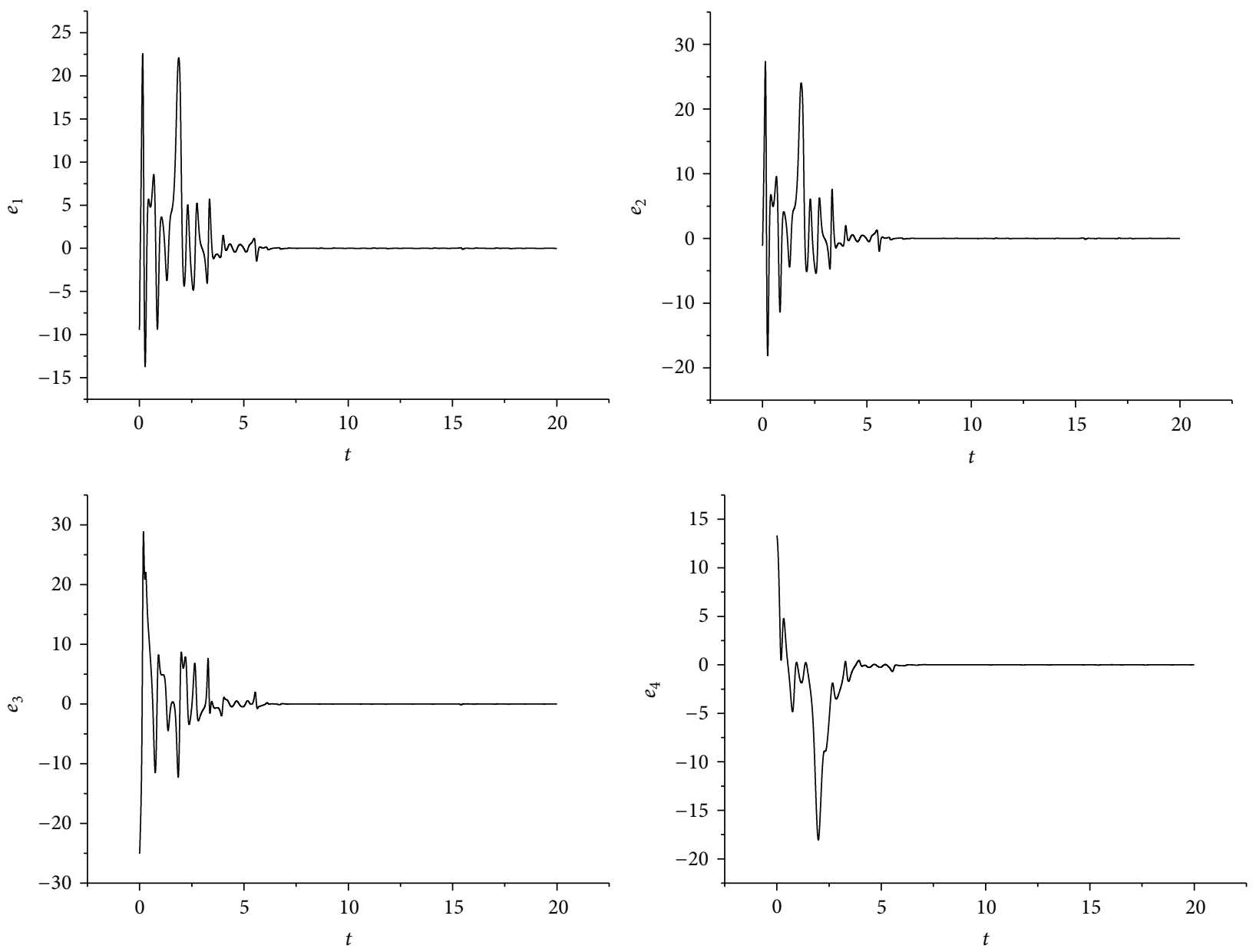

FIGURE 4: The synchronization errors of the state variables between the master system and the slave system.

one obtains $\lim _{t \rightarrow \infty}\left\|e_{i}\right\|=0 \quad(i=1,2,3,4)$. It means that the states of response system (3) and the states of drive system (2) are ultimately hybrid lag synchronized asymptotically.

\section{Numerical Simulations}

In this section, an illustrative example is presented to demonstrate the effectiveness of the proposed scheme. In the simulations, the system parameters are chosen as $a=$ $36, b=3, c=20, r_{1}=r_{2}=2$. The time delay is $\tau=1$. According to Theorem 1 , the initial conditions can be given as any values. For simplicity, the initial conditions of the master system and slave system are set to be $\left(x_{m}(0), y_{m}(0), z_{m}(0), w_{m}(0)\right)=(-2.0,3.0,0.4,0.2)$ and $\left(x_{s}(0), y_{s}(0), z_{s}(0), w_{s}(0)\right)=(1.2,0.3,0.5,0.6)$, respectively, and the feedback gain coefficients are supposed as $k_{i}=$ $k(i=1,2,3,4)$. From the theoretical analysis, it is known that conditions obtained analytically for the synchronization are only the sufficient conditions. Stronger feedback gain coefficient could induce synchronization easily, while smaller gain coefficient could not ensure the two chaotic systems reach hybrid lag synchronization Figure 2 shows the evolutions of the variables $x_{m}, y_{m}, z_{m}, w_{m}$ in drive system (2) and $x_{s}, y_{s}, z_{s}, w_{s}$ in response system (3) at $k=2.3$. The evolutions of the state variables $x_{m}(t-\tau), y_{m}(t-\tau), z_{m}(t-\tau), w_{m}(t-\tau)$ and $x_{s}, y_{s}, z_{s}, w_{s}$ are plotted in Figure 3 . Figure 4 depicts the synchronization errors of the state variables between the master system and the slave system. Figure 5 draws the identification of unknown parameters $a, b, c, r_{1}$, and $r_{2}$, from which it is obvious to see that the five unknown parameters converge to their real values, respectively. From the simulation results, we know that the proposed scheme works well and the system error states are regulated to zero asymptotically. Therefore, the hybrid synchronization of systems (2) and (3) can be achieved; that is, the third corresponding pair variables of systems (2) and (3) reach complete lag synchronization, while other three pair corresponding variables of systems (2) and (3) arrive lag antisynchronization.

\section{Conclusion}

In this paper, mixed lag synchronization in the hyperchaotic systems is studied. Considering the unknown system parameters, an adaptive control scheme has been proposed to ensure the hybrid lag synchronization between the drive and 

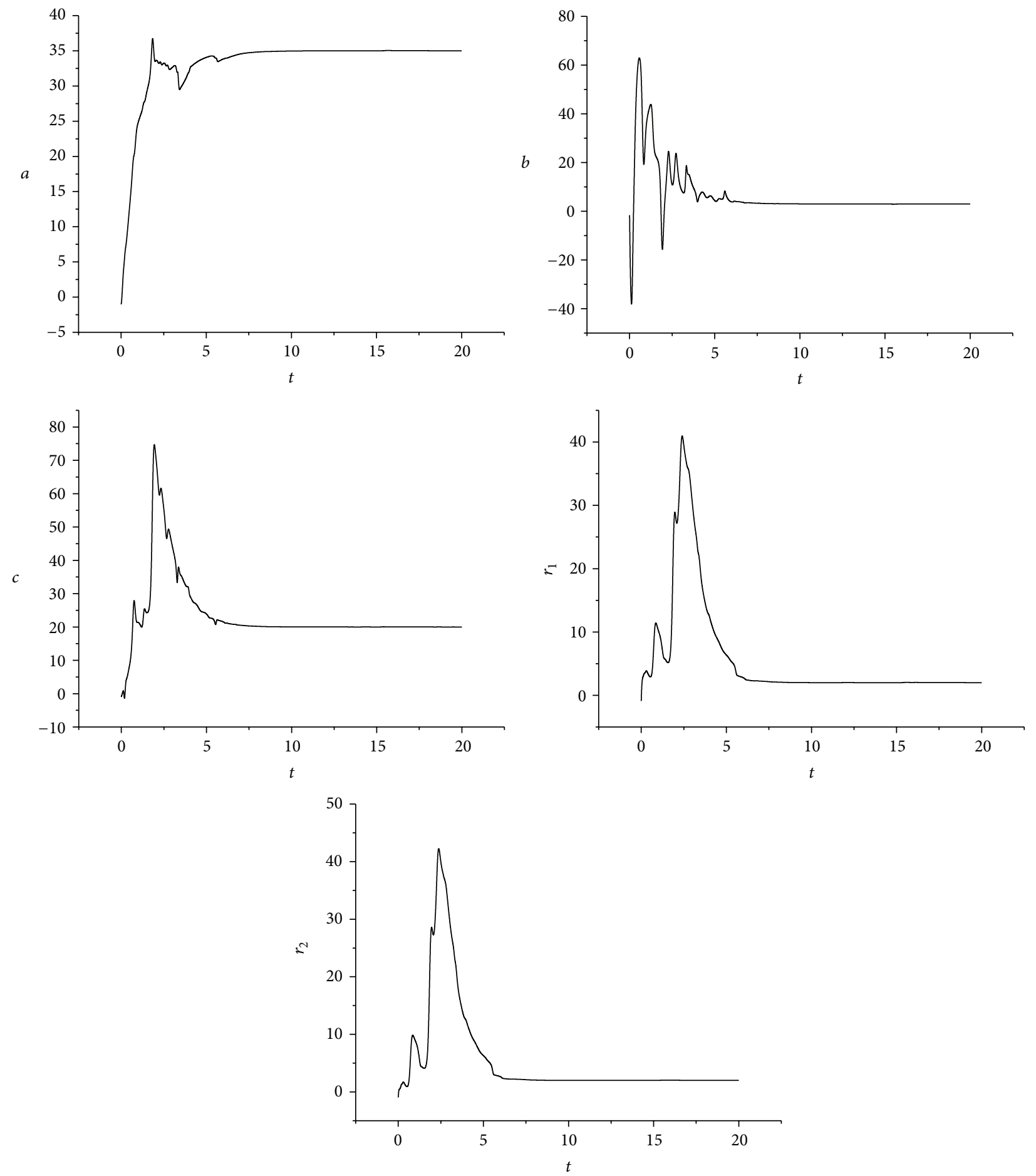

FIgURE 5: The identified results of the unknown parameters $a, b, c, r_{1}$, and $r_{2}$.

the controlled response systems. Numerical simulations have verified the effectiveness of the proposed technique.

\section{Conflict of Interests}

The authors declare that there is no conflict of interests regarding the publication of this paper.

\section{Acknowledgments}

This work was supported by National Natural Science Foundation of China (Grant no. 11102180, no. 61273106, and no. 61379064) and National Natural Science Foundation of the Jiangsu Higher Education Institutions of China (Grant no. BK2012672). 


\section{References}

[1] P. van Geert, Dynamic Systems of Development: Change between Complexity and Chaos, Harvester Wheatsheaf, 1994.

[2] S. Boccaletti, J. Kurths, G. Osipov, D. L. Valladares, and C. S. Zhou, "The synchronization of chaotic systems," Physics Report, vol. 366, no. 1-2, pp. 1-101, 2002.

[3] L. M. Pecora and T. L. Carroll, "Synchronization in chaotic systems," Physical Review Letters, vol. 64, no. 8, pp. 821-824, 1990.

[4] Q.-Y. Wang, Q.-S. Lu, and H.-X. Wang, "Transition to complete synchronization via near-synchronization in two coupled chaotic neurons," Chinese Physics, vol. 14, no. 11, pp. 2189-2195, 2005.

[5] Z.-M. Ge and C.-C. Chen, "Phase synchronization of coupled chaotic multiple time scales systems," Chaos, Solitons and Fractals, vol. 20, no. 3, pp. 639-647, 2004.

[6] C. Li, X. Liao, and K.-W. Wong, "Lag synchronization of hyperchaos with application to secure communications," Chaos, Solitons and Fractals, vol. 23, no. 1, pp. 183-193, 2005.

[7] J. Yang and G. Hu, “Three types of generalized synchronization," Physics Letters A: General, Atomic and Solid State Physics, vol. 361, no. 4-5, pp. 332-335, 2007.

[8] L. Wang, Z. Yuan, X. Chen, and Z. Zhou, "Lag synchronization of chaotic systems with parameter mismatches," Communications in Nonlinear Science and Numerical Simulation, vol. 16, no. 2, pp. 987-992, 2011.

[9] E. M. Shahverdiev, S. Sivaprakasam, and K. A. Shore, "Lag synchronization in time-delayed systems," Physics Letters A: General, Atomic and Solid State Physics, vol. 292, no. 6, pp. 320324, 2002.

[10] T.-Y. Chiang, J.-S. Lin, T.-L. Liao, and J.-J. Yan, "Antisynchronization of uncertain unified chaotic systems with dead-zone nonlinearity," Nonlinear Analysis: Theory, Methods and Applications, vol. 68, no. 9, pp. 2629-2637, 2008.

[11] M. Hu and Z. Xu, "A general scheme for Q-S synchronization of chaotic systems," Nonlinear Analysis: Theory, Methods and Applications, vol. 69, no. 4, pp. 1091-1099, 2008.

[12] Z.-L. Wang and X.-R. Shi, "Chaotic bursting lag synchronization of Hindmarsh-Rose system via a single controller," Applied Mathematics and Computation, vol. 215, no. 3, pp. 1091-1097, 2009.

[13] M. M. Al-Sawalha and M. S. M. Noorani, "Anti-synchronization of two hyperchaotic systems via nonlinear control," Communications in Nonlinear Science and Numerical Simulation, vol. 14, no. 8, pp. 3402-3411, 2009.

[14] S. Banerjee and A. R. Chowdhury, "Functional synchronization and its application to secure communications," International Journal of Modern Physics B, vol. 23, no. 9, pp. 2285-2295, 2009.

[15] M. Mossa Al-sawalha, M. S. M. Noorani, and M. M. Aldlalah, "Adaptive anti-synchronization of chaotic systems with fully unknown parameters," Computers and Mathematics with Applications, vol. 59, no. 10, pp. 3234-3244, 2010.

[16] J. Ma, Q.-Y. Wang, W.-Y. Jin, and Y.-F. Xia, "Control chaos in Hindmarsh-Rose neuron by using intermittent feedback with one variable," Chinese Physics Letters, vol. 25, no. 10, pp. 3582$3585,2008$.

[17] Z. $\mathrm{Li}$ and D. Xu, "A secure communication scheme using projective chaos synchronization," Chaos, Solitons and Fractals, vol. 22 , no. 2, pp. 477-481, 2004.
[18] A. N. Njah, "Tracking control and synchronization of the new hyperchaotic Liu system via backstepping techniques," Nonlinear Dynamics, vol. 61, no. 1-2, pp. 1-9, 2010.

[19] J. H. Park, "Synchronization of Genesio chaotic system via backstepping approach," Chaos, Solitons and Fractals, vol. 27, no. 5, pp. 1369-1375, 2006.

[20] J. Ma, W.-T. Su, and J.-Z. Gao, "Optimization of selfadaptive synchronization and parameters estimation in chaotic Hindmarsh-Rose neuron model," Acta Physica Sinica, vol. 59, no. 3, pp. 1554-1561, 2010.

[21] C.-F. Feng, Y. Zhang, J.-T. Sun, W. Qi, and Y.-H. Wang, "Generalized projective synchronization in time-delayed chaotic systems," Chaos, Solitons and Fractals, vol. 38, no. 3, pp. 743-747, 2008 .

[22] J. Ma, F. Li, L. Huang, and W.-Y. Jin, "Complete synchronization, phase synchronization and parameters estimation in a realistic chaotic system," Communications in Nonlinear Science and Numerical Simulation, vol. 16, no. 9, pp. 3770-3785, 2011.

[23] Z. Wang, "Chaos synchronization of an energy resource system based on linear control," Nonlinear Analysis: Real World Applications, vol. 11, no. 5, pp. 3336-3343, 2010.

[24] C. K. Volos, I. M. Kyprianidis, and I. N. Stouboulos, "Various synchronization phenomena in bidirectionally coupled double scroll circuits," Communications in Nonlinear Science and Numerical Simulation, vol. 16, no. 8, pp. 3356-3366, 2011.

[25] A. A. Selivanov, J. Lehnert, T. Dahms, P. Hövel, A. L. Fradkov, and E. Schöll, "Adaptive synchronization in delay-coupled networks of Stuart-Landau oscillators," Physical Review E: Statistical, Nonlinear, and Soft Matter Physics, vol. 85, no. 1, Article ID 016201, 2012.

[26] D. Ghosh, A. R. Chowdhury, and P. Saha, "Multiple delay Rössler system-Bifurcation and chaos control," Chaos, Solitons and Fractals, vol. 35, no. 3, pp. 472-485, 2008.

[27] J.-H. Chen, H.-K. Chen, and Y.-K. Lin, "Synchronization and anti-synchronization coexist in Chen-Lee chaotic systems," Chaos, Solitons and Fractals, vol. 39, no. 2, pp. 707-716, 2009.

[28] S. K. Bhowmick, C. Hens, D. Ghosh et al., "Mixed synchronization in chaotic oscillators using scalar coupling," Physics Letters A: General, Atomic and Solid State Physics, vol. 376, no. 36, pp. 2490-2495, 2012.

[29] Q. Zhang, J. Lü, and S. Chen, "Coexistence of anti-phase and complete synchronization in the generalized Lorenz system," Communications in Nonlinear Science and Numerical Simulation, vol. 15, no. 10, pp. 3067-3072, 2010.

[30] M.-C. Ho, Y.-C. Hung, and C.-H. Chou, "Phase and anti-phase synchronization of two chaotic systems by using active control," Physics Letters A: General, Atomic and Solid State Physics, vol. 296, no. 1, pp. 43-48, 2002.

[31] S. Pang and Y. Liu, "A new hyperchaotic system from the Lü system and its control," Journal of Computational and Applied Mathematics, vol. 235, no. 8, pp. 2775-2789, 2011. 

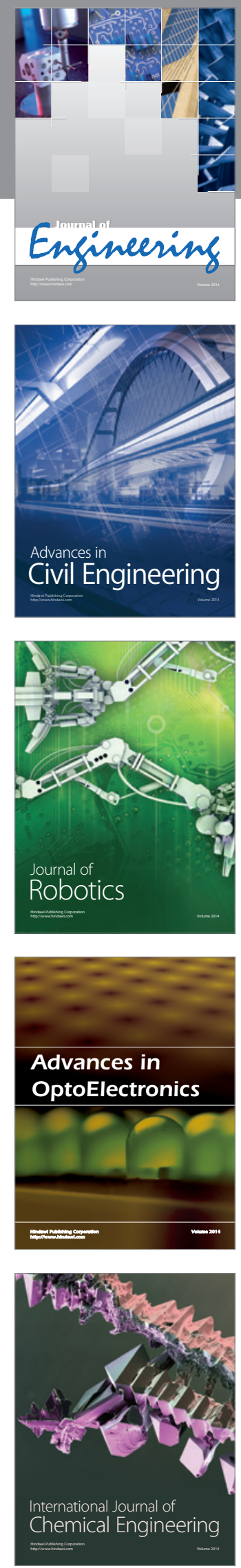

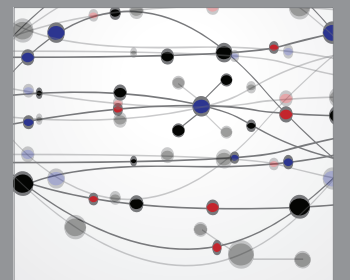

The Scientific World Journal
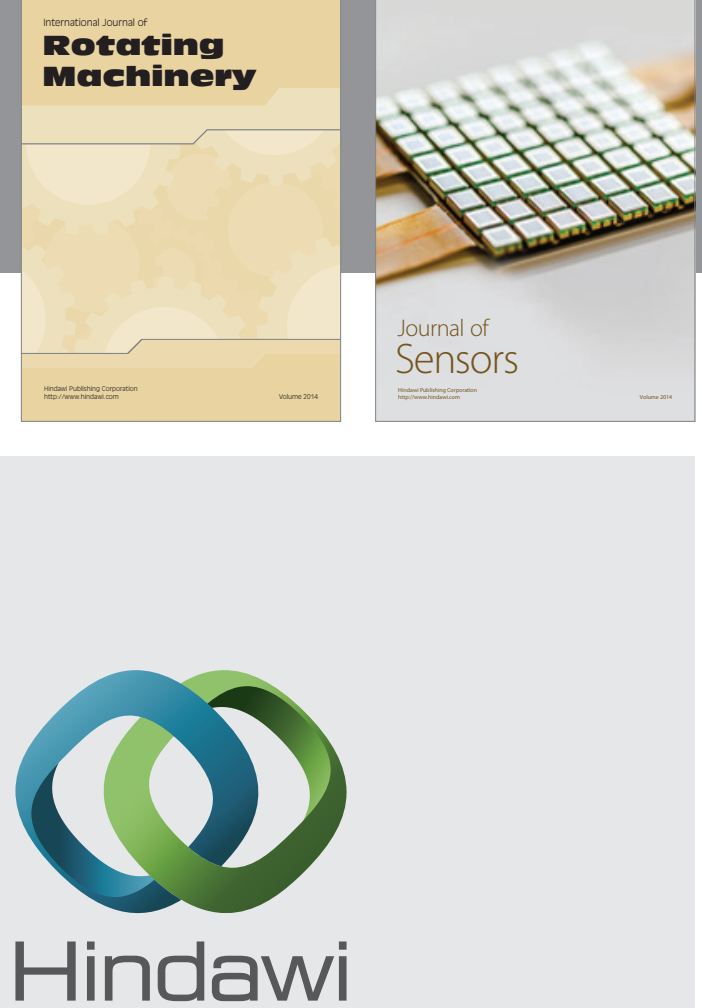

Submit your manuscripts at http://www.hindawi.com
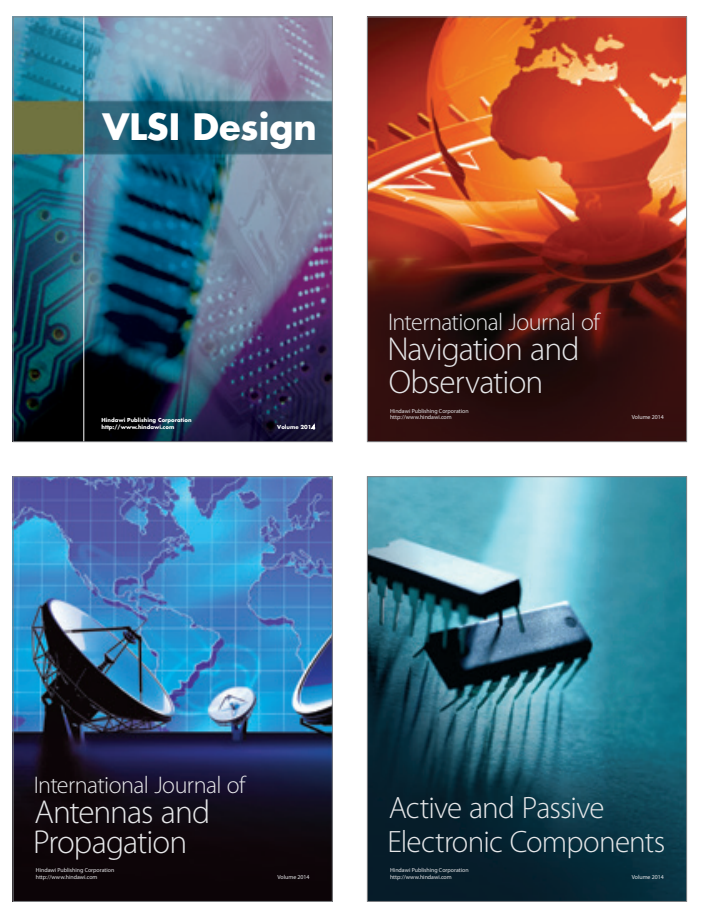
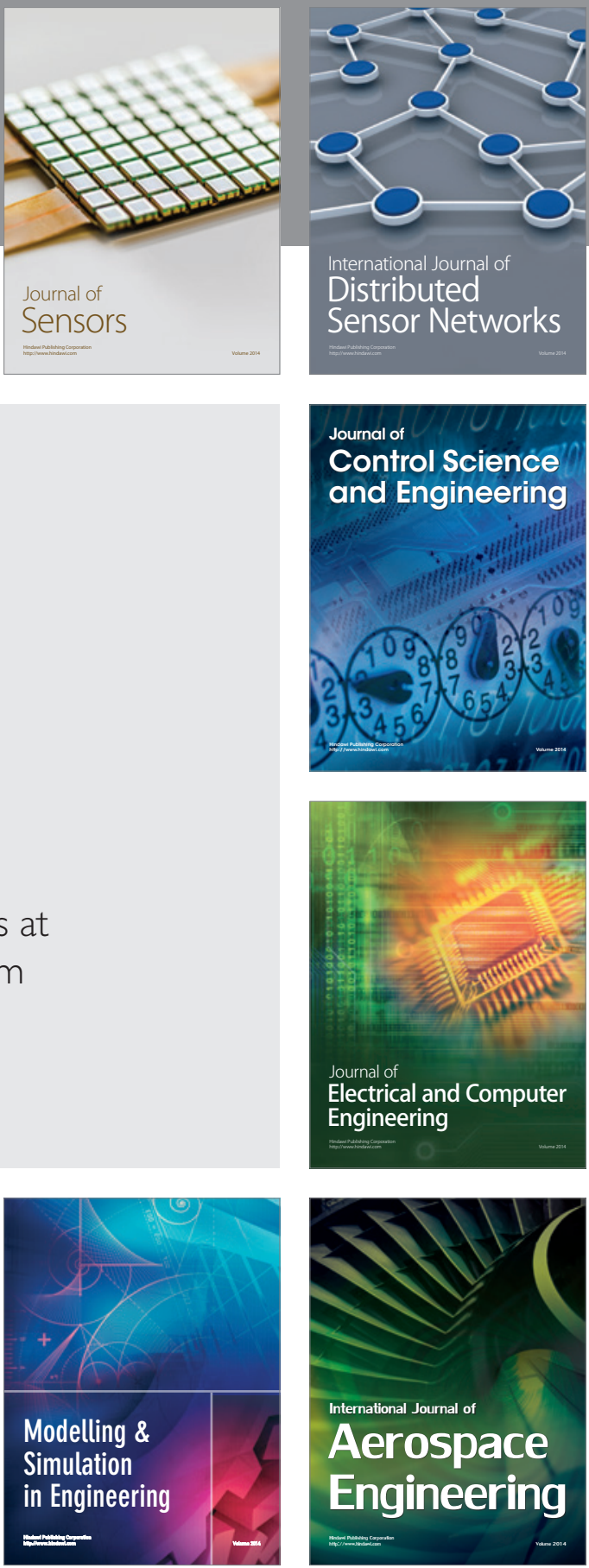

Journal of

Control Science

and Engineering
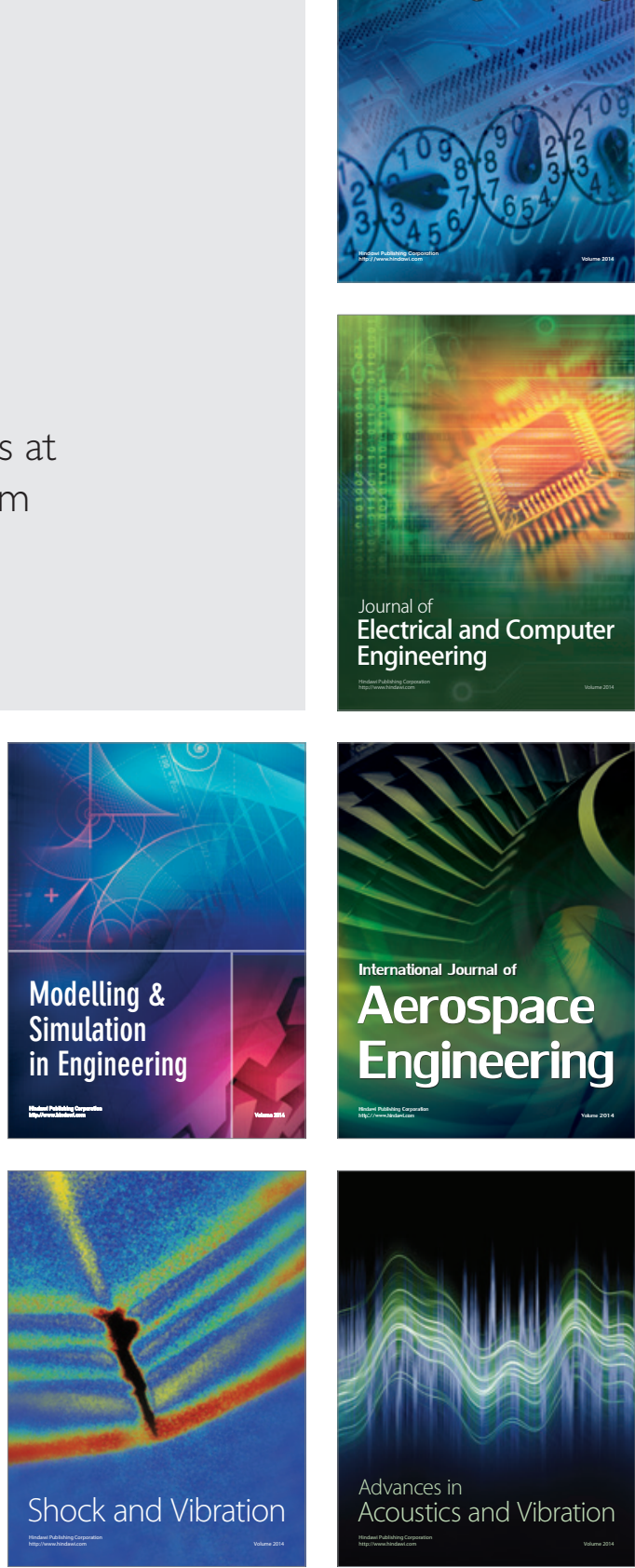